\title{
Fatal Outcome in a Kidney-Pancreas Transplant Recipient With COVID-19
}

\author{
Kulachanya Suwanwongse ${ }^{1}$, Nehad Shabarek ${ }^{1}$ \\ 1. Internal Medicine, Lincoln Medical Center, New York City, USA
}

Corresponding author: Kulachanya Suwanwongse, kulachanya.suwanwongse@gmail.com

\begin{abstract}
Severe acute respiratory syndrome coronavirus 2 (SARS-CoV-2) is a highly contagious pathogen causing the novel coronavirus disease 2019 (COVID-19), the ongoing unprecedented pandemic in 2020. SARS-CoV-2 primarily targets the respiratory systems, so acute respiratory distress syndrome is the major cause of death. Clinical courses of COVID-19 are variable and unpredictable, while some epidemiologic and clinical factors have been found to have a negative impact on the disease prognosis. Despite a growing report on clinical characteristics and prognosis of patients with COVID-19, the data in the special population, including transplant recipients, is still limited. Herein we report on the clinical features and fatal outcome of COVID19 in a dual pancreas-kidney transplant recipient (with failure of the pancreas graft). Our case illustrates the similarities and differences of the COVID-19 disease course between transplant recipients and the general population. We proposed that the pre-existing T-cell dysfunction from the long-term use of immunosuppressive agents in organ transplant recipients adversely affects COVID-19 prognosis and worsens COVID-19 mortality.
\end{abstract}

Categories: Internal Medicine, Allergy/Immunology, Infectious Disease

Keywords: novel corona virus, transplant, renal failure, case report, covid-19, immunocompromised, immunosuppesive

\section{Introduction}

The novel coronavirus disease 2019 (COVID-19), caused by severe acute respiratory syndrome coronavirus 2 (SARS-CoV-2), is one of the greatest pandemic challenges in the 21st century. Since its first report in December 2019, SARS-CoV-2 has rapidly spread worldwide resulting in over five million confirmed cases and almost 400,000 deaths [1].

In general, transplant recipients are vulnerable to develop serious infections due to chronic medical-induced immunosuppressive states. However, impaired immune functions may paradoxically protect transplant patients from the hyper-inflammatory response to SARS-CoV-2 and thus dampen the disease severity. Previous reports on the outcomes of COVID-19 in transplant recipients showed contradictory results, in which the mortality rate ranges from $0 \%$ to $67 \%$ [2-9]. The diversity in COVID-19 prognoses across each literature may be due to different baseline patient characteristics, including age, sex, and comorbidities, as well as various management strategies across institutions. Herein we report our experience in treating COVID-19 in a dual pancreas-kidney transplant recipient and discuss the current knowledge regarding COVID-19 in transplant patients.

() Copyright 2020 Suwanwongse et al. This is an open access article distributed under the terms of the Creative Commons Attribution License CC-BY 4.0., which permits unrestricted use, distribution, and reproduction in any medium, provided the original author and source are credited.

\section{Case Presentation}

We present the case of a 52-year-old male who received dual pancreas-kidney transplantation from a deceased donor 20 years ago because of end-stage renal disease (ESRD) due to diabetic nephropathy. He had pancreas graft failure and underwent second pancreas transplantation 12 years ago with immediate graft failure from thrombosis and third pancreas transplantation 11 years ago with failure of the graft a year later due to viral infection. He then decided not to have further attempts of pancreas transplantation.

His maintenance immunosuppressive therapy included oral tacrolimus (TAC) 4 mg twice daily, mycophenolate mofetil (MMF) $250 \mathrm{mg}$ twice daily, and prednisone $5 \mathrm{mg}$ once daily. He reported medication compliance and was adherent to follow-up appointments. His baseline creatinine ranged $2.0-2.5 \mathrm{mg} / \mathrm{dl}$ over the past year. The decline in his renal function was due to diabetic nephropathy, which was diagnosed by renal biopsy. His most recent serum creatinine ( $\mathrm{Cr}$ ), two months prior to the current admission, was 2.34 $\mathrm{mg} / \mathrm{dl}$. The patient also had insulin-dependent diabetes mellitus (recent hemoglobin A1C 7.8\%), hypertension, and obesity (body mass index $30.18 \mathrm{~kg} / \mathrm{m}^{2}$ ), for which he took losartan $50 \mathrm{mg}$ once daily, metoprolol succinate $25 \mathrm{mg}$ once daily, rosuvastatin $5 \mathrm{mg}$ once daily, and insulin regimens.

He presented with worsening dyspnea for three days and reported subjective fever, dry cough, watery diarrhea, polydipsia, and polyuria. On initial evaluation, his temperature was $97.8^{\circ} \mathrm{F}$, blood pressure was $145 / 78 \mathrm{mmHg}$, heart rate was 112 beats per minute, and respiratory rate was 28 breaths per minute with 


\section{Cureus}

oxygen saturation $\left(\mathrm{SpO}_{2}\right)$ of $92 \%$ on room air. He had dry mucous membranes and did not appear to use accessory respiratory muscles. His lung examination revealed rales in both lungs. His neurological examination was normal. Chest x-ray (CXR) showed bilateral multifocal ground-glass appearances (Figure 1). Laboratory values are described in Table 1.

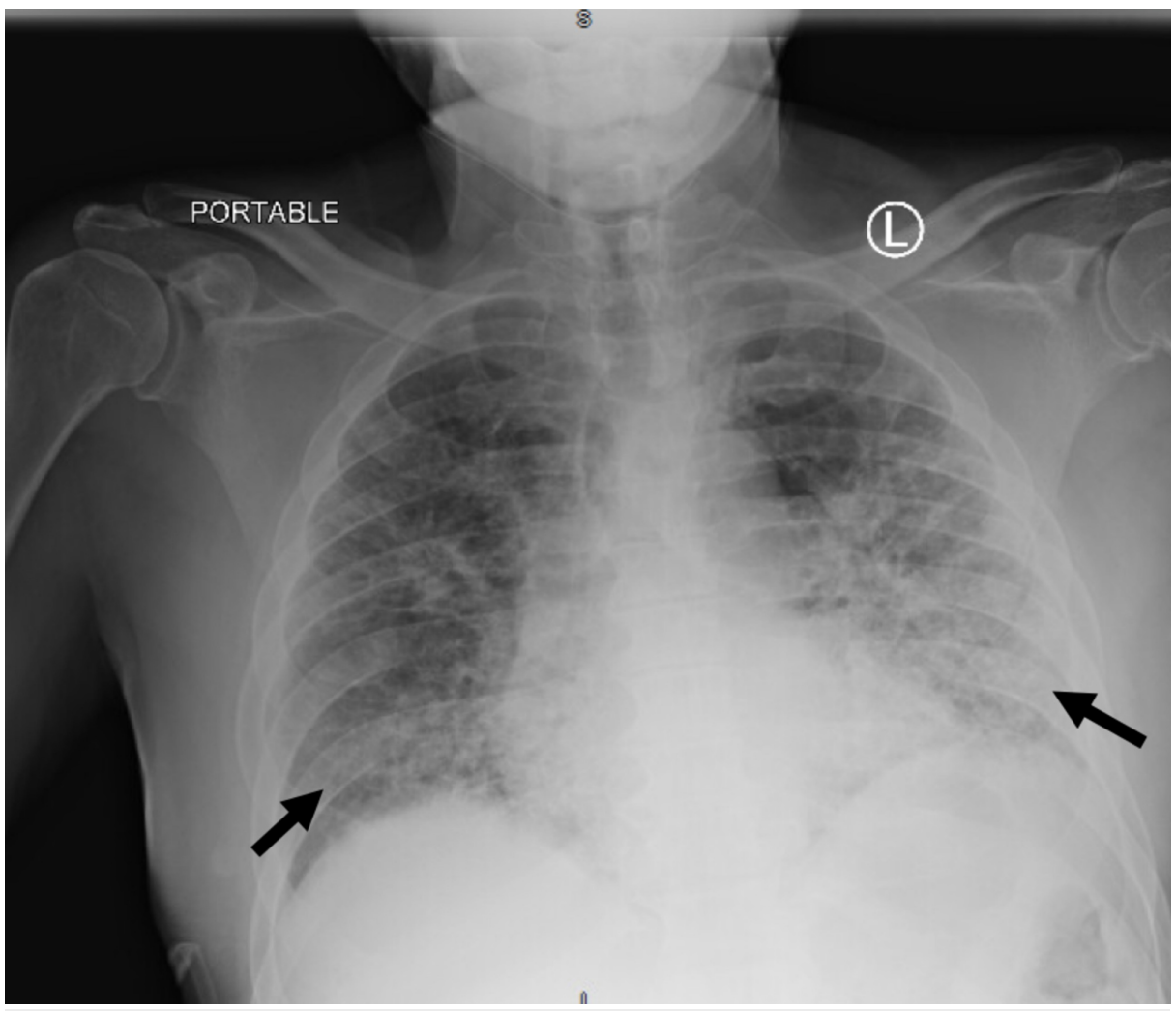

FIGURE 1: Chest x-ray showing bilateral multifocal ground-glass appearances

\section{Laboratory results}

Leukocytosis

Lymphopenia

Neutrophilia

Creatinine

Hypokalemia

\section{Acidemia}

Hepatic function panel

Inflammatory markers significantly elevated
White blood cell count $12,200 / \mathrm{mcl}$

Lymphocytes 5.9\%

Neutrophils $85.7 \%$

$1.54 \mathrm{mg} / \mathrm{dl}$

Potassium $2.7 \mathrm{mmol} / \mathrm{l}$

Venous blood gas $\mathrm{pH} 7.26, \mathrm{pCO}_{2} 41.7 \mathrm{mmHg}$

Normal

C-reactive protein 9.22 mg/l, D-dimer 996 ng/ml, ferritin 569 ng/ml

Ketonemia (beta-hydroxybutyrate $>4.40 \mathrm{mmol} / \mathrm{l}$, normal $<0.27 \mathrm{mmol} / \mathrm{l}$ ) and ketonuria

\section{TABLE 1: Initial laboratory results of the patient}

SARS-CoV-2 reverse transcriptase-polymerase chain reaction (RT-PCR) from the nasopharyngeal swab specimen was positive. He was diagnosed with diabetic ketoacidosis (DKA) and COVID-19. Due to a lack of a negative-pressure room in our institution, he was admitted to a regular patient room on the medicine floor, with droplet and contact precaution. The patient received the standard treatment of DKA, including insulin drip, aggressive electrolytes replacement, and intravenous hydration. He received supplemental oxygen via 
nasal cannula (4 1/min), and his $\mathrm{SpO}_{2}$ was improved to $99 \%-100 \%$. Hydroxychloroquine (400 $\mathrm{mg}$ twice daily on the first day, and $200 \mathrm{mg}$ twice daily for the following four days) was administered for COVID-19 treatment as per hospital protocol. Assuming his immunocompromised status, the primary team decided to give antibiotics: oral doxycycline and intravenous ceftriaxone for possible secondary bacterial pneumonia. His TAC was stopped. MMF and prednisone were continued with the same dosage. His blood pressure medications and statin were discontinued.

The next day, his DKA was resolving. However, he developed acute hypoxemic respiratory failure requiring invasive ventilation. His arterial oxygen partial pressure $\left(\mathrm{PaO}_{2}\right)$ to fractional inspired oxygen $\left(\mathrm{FiO}_{2}\right)(\mathrm{P}: \mathrm{F})$ ratio was 69 indicating severe acute respiratory distress syndrome (ARDS). Blood tests showed serum creatinine (Cr) rising to $2.49 \mathrm{mg} / \mathrm{dl}$. He was transferred to our COVID-19 ventilator unit for intensive care. On Day 3, he developed septic shock requiring small doses of intravenous norepinephrine drip (range 2-10 $\mathrm{mcg} / \mathrm{min}$ ). His prednisone was discontinued, and he was started on stress dose corticosteroids: intravenous hydrocortisone $50 \mathrm{mg}$ every six hours.

On Day 5, his Cr worsened to $3.38 \mathrm{mg} / \mathrm{dl}$, while his respiratory function remained stable. The primary team contacted his transplant center for possible transfer due to suspected acute graft rejection from discontinuation of TAC. However, he was rejected due to a lack of patient beds. TAC was re-started. The next day, the patient became more hypoxemic with a P:F ratio of 54 . $\mathrm{His}_{\mathrm{SpO}_{2}}$ was around $80 \%$ despite adjusted high ventilator setting: volume control - positive end-expiratory pressure (PEEP) value of 20 $\mathrm{mmHg}$ and $100 \% \mathrm{FiO}_{2}$. CXR showed worsening pneumonia and ARDS. He was started on the proning protocol, with mild improvement of $\mathrm{SpO}_{2}$ to $85 \%-88 \%$. Although his hypoxemia was likely from severe ARDS, the primary team decided to give enoxaparin to treat possible pulmonary embolism, assuming the medication risks were lower than benefits.

On admission day 8 , he acutely developed oliguria with urine output less than $50 \mathrm{ml}$ in eight hours. His creatinine was doubled to $6.45 \mathrm{mg} / \mathrm{dl}$. His transplant center was contacted again, but there was still no bed available. The daily changes in his creatinine are demonstrated in Figure 2. He received hemodialysis with total ultrafiltration of 21 . However, his conditions were progressively worsening and he eventually died on Day 11. Multiple blood and sputum cultures were done during hospital courses, which all revealed negative results. His cause of death was septic shock and multi-organ failure from severe COVID-19.

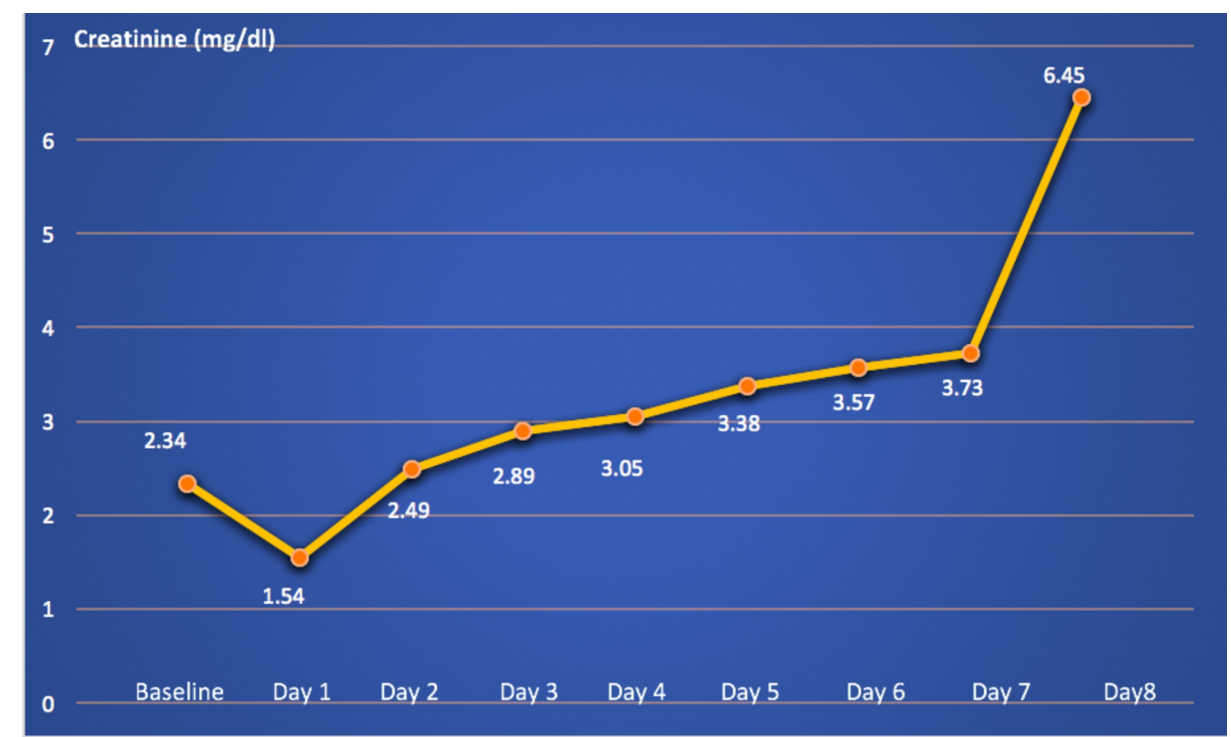

FIGURE 2: Changes in patient's creatinine level during hospitalization

\section{Discussion}

SARS-CoV-2 uses angiotensin-converting enzyme 2 (ACE2) receptors to enter the host cells causing a spectrum of diseases ranging from flu-like illness, pneumonia to severe ARDS, septic shock, and death $[10,11]$. The uncontrolled hyper-inflammatory response to SARS-CoV-2, rather than the own viral virulence, presumably underlies the COVID-19 severity $[12,13]$. Certain comorbidities, including hypertension, diabetes mellitus, and cardiovascular diseases, adversely impact COVID-19 prognosis, probably due to the changes in the renin-angiotensin system (RAS) or underlying chronic inflammatory states [11,14]. Many research teams have been repurposing various anti-viral and immunomodulating agents to treat COVID-19. However, to date, no specific drugs have proven mortality benefits, and unfortunately, the best COVID-19 management is supportive measures, which include the provision of supplemental oxygen, invasive 
ventilation, and extracorporeal membrane oxygenation [15].

Long-term immunosuppressive therapy in organ transplant recipients may alter clinical features and outcomes of COVID-19. However, considering our patient and previously published literature, transplant recipients with COVID-19 did not show prominent differences in terms of clinical symptoms, laboratory values, and CXR findings [2-9].

Our patient had hypertension, diabetes mellitus, and obesity, which are identified as poor prognostic markers of COVID-19 in the normal population [11,14]. Whether these comorbidities negatively impact COVID-19 in transplant recipients is not well-characterized, but most of the literature published so far and our case report suggest a similar result [2-9]. Recent evidence showed that the duration of organ transplantation correlates with COVID-19 severity [16]. Our patient underwent dual kidney-pancreas transplantation for 20 years, so he had unfavorable outcomes leading ultimately to death. Whether the type and number of organ transplantation affect the COVID-19 courses remains undetermined.

The long-term use of immunosuppressive medications in organ transplant recipients is associated with the decrease in T-cell number and function; TAC and MMF preferentially inhibit T-cell response. Tcell lymphocytopenia has also been found in patients with COVID-19 and is correlated with the disease severity [17]. Whether the chronic T-cell suppression in organ transplant recipients attenuates the clinical course and severity of COVID-19 remains unknown. Recently, we reported the extremely high mortality rate of HIV patients with SARS-CoV-2 infection [18]. T-cell impairment is the major characteristic of HIVassociated immunopathology. Based on our observations, we propose that the previous medically induced Tcell impairment negatively affects COVID-19 prognoses in the transplant recipients. Further research exploring the role of T-cell lymphocytes in SARS-CoV-2 pathogenesis will be worthwhile.

It is uncertain whether the immunosuppressive agents should be adjusted in transplant recipients who have been infected with SARS-CoV-2. Aberrant host-immune reactions characterized by hyper-inflammatory states and persistent cytokines releases likely contributed to COVID-19 severity. Severe COVID-19 patients had considerably high serum pro-inflammatory cytokines, including tumor necrosis factor (TNF)- $\alpha$, interleukin (IL)-1, and IL-6 [13]. Abrupt discontinuation of immunosuppressive agents may lead to an exaggerated rebound inflammatory response to SARS-CoV-2. A study from the Netherlands found that immunosuppressive drug withdrawal worsened COVID-19 mortality. However, in this report, immunosuppressive agents were discontinued in patients with severe disease, presumably with high mortality risks. The current expert opinion urges adjusting the immunosuppressive agents based on COVID19 severity. We have summarized the recommendations in Table 2 [19].

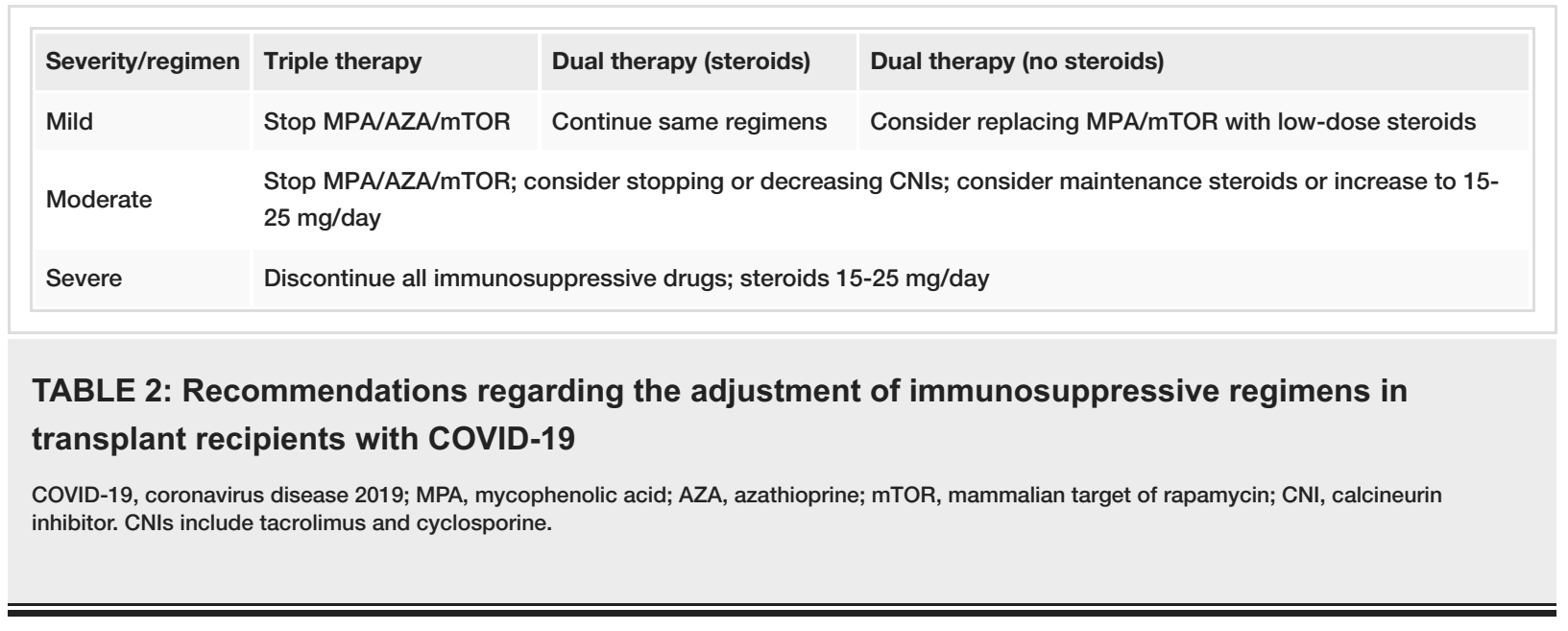

Surprisingly, on admission, our patient had an increase in the glomerular infiltration rate. This is likely due to the early phase of sepsis and acute kidney injury (AKI). Clinicians should be aware that patients may have lower serum $\mathrm{Cr}$ during the early phase of AKI. Other common causes of the acute drop in $\mathrm{Cr}$ include urinary tract obstruction and certain medication effects. The doubled rise of his $\mathrm{Cr}$ level on Day 8 was likely due to septic shock and multi-organ failure. The patient was unlikely to have acute renal graft rejection from TAC withdrawal as he had undergone transplantation for more than 10 years and TAC was discontinued for only a few days. The definitive diagnosis of acute renal graft rejection requires kidney biopsy. However, it was not done in our patient as the diagnosis would not change our management. The patient required hemodialysis as he had oliguric acute renal failure and suffered from severe COVID-19.

\section{Conclusions}

We report a clinical course and lethal outcome of COVID-19 in a transplant recipient. Pre-existing T-cell immune response deficits from long-term use of immunosuppressive agents may worsen the prognosis of 
COVID-19 in transplant recipients. The optimal management of COVID-19 in the post-transplant population remains unclear. The recent expert opinion recommended adjusting immunosuppressive agents based on COVID-19 severity. There is an urgent need to develop the collaborative management guidelines of organ transplant recipients with COVID-19 in the non-transplant centers to provide patients the best available care.

\section{Additional Information \\ Disclosures}

Human subjects: Consent was obtained by all participants in this study. Conflicts of interest: In compliance with the ICMJE uniform disclosure form, all authors declare the following: Payment/services info: All authors have declared that no financial support was received from any organization for the submitted work. Financial relationships: All authors have declared that they have no financial relationships at present or within the previous three years with any organizations that might have an interest in the submitted work. Other relationships: All authors have declared that there are no other relationships or activities that could appear to have influenced the submitted work.

\section{References}

1. Coronavirus disease 2019 (COVID-19): situation report - 132 . (2020). Accessed: May 31, 2020: https://www.who.int/docs/default-source/coronaviruse/situation-reports/20200531-covid-19-sitrep132.pdf?sfvrsn=d9c2ea....

2. Fernández-Ruiz M, Andrés A, Loinaz C, et al.: COVID-19 in solid organ transplant recipients: a single center case series from Spain [Epub ahead of print]. Am J Transplant. 2020, 10.1111/ajt.15929

3. Zhu L, Xu X, Ma K, et al.: Successful recovery of COVID-19 pneumonia in a renal transplant recipient with long-term immunosuppression [Epub ahead of print]. Am J Transplant. 2020, 10.1111/ajt.15869

4. Akalin E, Azzi Y, Bartash R, et al.: Covid-19 and kidney transplantation. N Engl J Med. 2020, 382:2475-2477. 10.1056/NEJMc2011117

5. Pereira MR, Mohan S, Cohen DJ, et al.: COVID-19 in solid organ transplant recipients: initial report from the US epicenter [Epub ahead of print]. Am J Transplant. 2020, 10.1111/ajt.15941

6. Bhoori S, Rossi RE, Citterio D, Mazzaferro V: COVID-19 in long-term liver transplant patients: preliminary experience from an Italian transplant centre in Lombardy. Lancet Gastroenterol Hepatol. 2020, 5:532-533. 10.1016/S2468-1253(20)30116-3

7. Fung M, Chiu CY, DeVoe C, et al.: Clinical outcomes and serologic response in solid organ transplant recipients with COVID-19: a case series from the United States [Epub ahead of print]. Am J Transplant. 2020, 10.1111/ajt.16079

8. Hoek RAS, Manintveld OC, Betjes MGH, et al.: Covid-19 in solid organ transplant recipients: a single center experience [Epub ahead of print]. Transpl Int. 2020, 10.1111/tri.13662

9. Gao F, Zheng KI, Gu JY, George J, Zheng MH: COVID-19 and liver transplantation: lessons learned from three reported cases [Epub ahead of print]. Transpl Infect Dis. 2020, e13335. 10.1111/tid.13335

10. Lu R, Zhao X, Li J, et al.: Genomic characterisation and epidemiology of 2019 novel coronavirus: implications for virus origins and receptor binding [Epub ahead of print]. Lancet. 2020, 395:565-574. 10.1016/S0140-6736(20)30251-8

11. Zhou F, Yu T, Du R, et al.: Clinical course and risk factors for mortality of adult inpatients with COVID-19 in Wuhan, China: a retrospective cohort study. Lancet. 2020, 395:1054-1062. 10.1016/S01406736(20)30566-3

12. Giamarellos-Bourboulis EJ, Netea MG, Rovina N, et al.: Complex immune dysregulation in COVID-19 patients with severe respiratory failure. Cell Host Microbe. 2020, 27:992-1000.e3. 10.1016/j.chom.2020.04.009

13. Qin C, Zhou L, Hu Z, et al.: Dysregulation of immune response in patients with coronavirus 2019 (COVID19) in Wuhan, China [Epub ahead of print]. Clin Infect Dis. 2020, ciaa248. 10.1093/cid/ciaa248

14. Wu C, Chen X, Cai Y, et al.: Risk factors associated with acute respiratory distress syndrome and death in patients with coronavirus disease 2019 pneumonia in Wuhan, China [Epub ahead of print]. JAMA Intern Med. 2020, e200994. 10.1001/jamainternmed.2020.0994

15. Nicola M, O'Neill N, Sohrabi C, Khan M, Agha M, Agha R: Evidence based management guideline for the COVID-19 pandemic - review article. Int J Surg. 2020, 77:206-216. 10.1016/j.ijsu.2020.04.001

16. Merli M, Perricone G, Lauterio A, et al.: Reply to "Coronaviruses and immunosuppressed patients. The facts during the third epidemic" [Epub ahead of print]. Liver Transpl. 2020, 10.1002/1t.25806

17. Xu B, Fan CY, Wang AL, et al.: Suppressed T cell-mediated immunity in patients with COVID- 19: a clinical retrospective study in Wuhan, China. J Infect. 2020, 81:e51-e60. 10.1016/j.jinf.2020.04.012

18. Suwanwongse K, Shabarek N: Clinical features and outcome of HIV/SARS-CoV-2 co-infected patients in the Bronx, New York City [Epub ahead of print]. J Med Virol. 2020, 10.1002/jmv.26077

19. Maggiore U, Abramowicz D, Crespo M, et al.: How should I manage immunosuppression in a kidney transplant patient with COVID-19? An ERA-EDTA DESCARTES expert opinion. Nephrol Dial Transplant. 2020, 35:899-904. 10.1093/ndt/gfaa130 